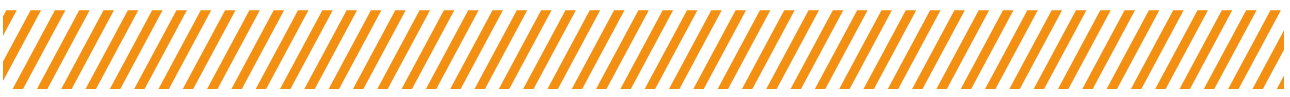

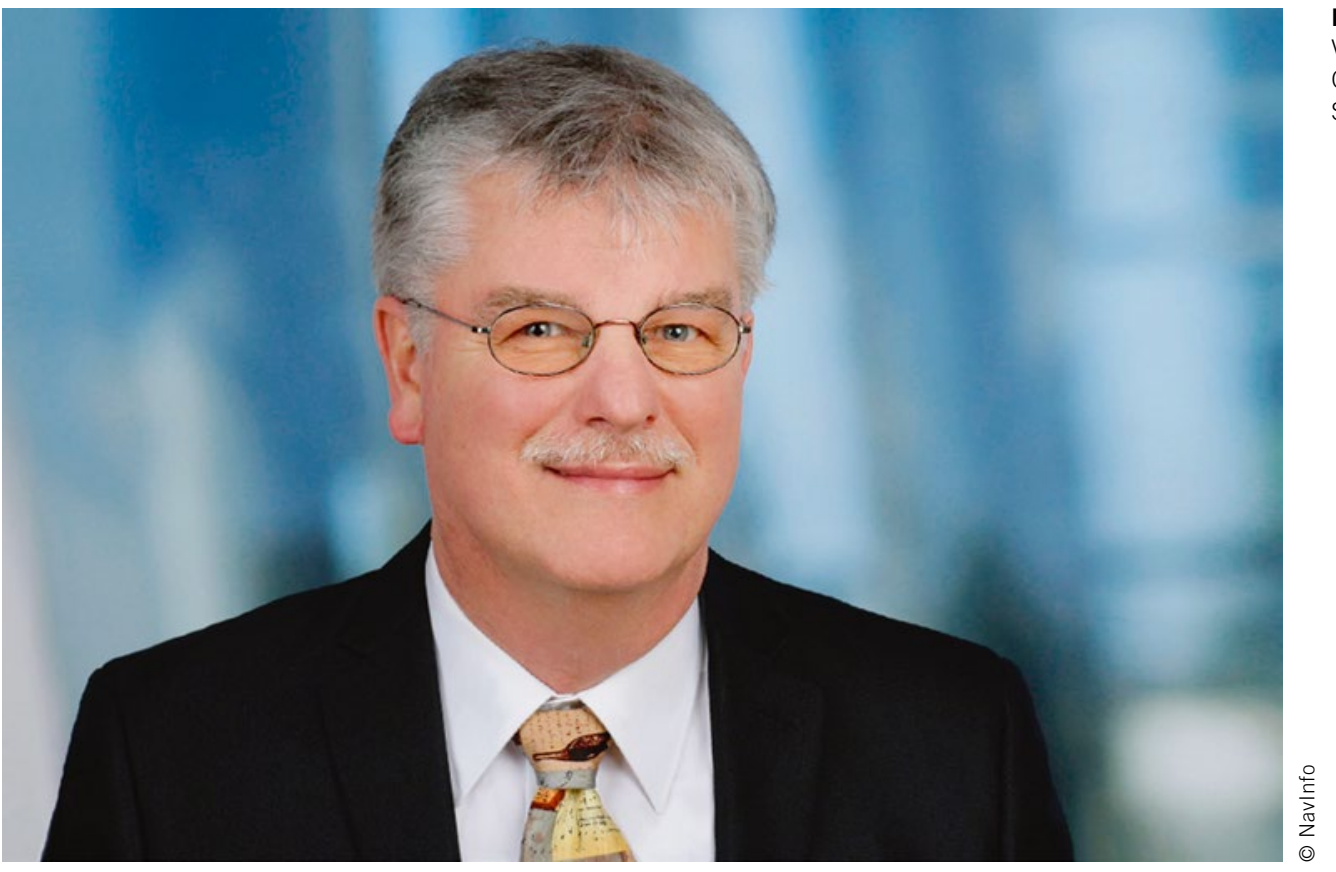

Dr. Volker Sasse

Vice President NavInfo,

Chairman NDS e.V.

Shanghai (China)

\section{Autonomous Driving}

\section{From Individualism towards Collectivism}

The automobile industry has traditionally taken a highly individualist approach. The manufacturers have concentrated on conducting their own research and outfitted their cars with their own systems. However, as the importance of the driverless vehicle continues to rise, we can expect to see more and more manufacturers willing to adopt a collectivist approach. Driverless vehicles will no longer navigate our roadways as independent units, and their control will no longer be bound to an internal array of sensors. Instead, they will interact with global systems, using their sensors to record changes in traffic conditions, relaying the corresponding data and also receiving data generated elsewhere. A cloud-based exchange of such information like real-time updating of digital maps will be indispensable for securing and improving the safety and effectiveness of driverless vehicles.

The compilation and sharing of information in a unified and standardised system is a prerequisite for the smooth and safe operation of self-driving vehicles. It follows that if autonomous vehicles are to be made available to consumers on a mass-market scale, car manufacturers, automotive systems suppliers and tech companies will be forced to open their systems and work together in the context of a mutually beneficial syndicate. After all, sufficiently precise information will depend on a large of independent generators of high quality data. This is why it will be crucial for all vehicles, no matter what the manufacturer, to store and administer their data in a common cloud and not in any number of separate clouds.

In this regard, there is simply no alternative to standardisation. We have taken an important first step to promoting the level of cooperation that is necessary by establishing the Germany-based Navigation Data Standard Association (NDSA). The aim of the NDSA, whose current members include more than 30 international automobile manufacturers, automotive systems suppliers and mapmakers, is to maintain a Navigation Data Standard (NDS) for digital maps in infotainment systems. In order to meet the challenges of the future, we have expanded the existing international standard so as to enable the representation of the necessary high-precision maps. In 2015, the Navigation Data Standard Association also launched its Open Auto Drive Forum (OADF), an exchange platform that gives companies and researchers an opportunity to synchronise their solutions.

The development of autonomous vehicle technology can be expected to go hand in hand with a shift away from individualism and toward collectivism. We will no longer identify ourselves as drivers of a vehicle, but as passengers in collective means of mobility. It will continue to be important for all of the stakeholders involved to internalise this notion of collectivism so as to better enable the success of a promising revolution in our mobile world. 